\title{
Le double portrait et les trois oranges : effets de miroir dans Shamrock Tea de Ciaran Carson
}

\section{Elisabeth Delattre}

\section{Q OpenEdition \\ 1 Journals}

\section{Édition électronique}

URL : http://journals.openedition.org/etudesirlandaises/3037

DOI : 10.4000/etudesirlandaises.3037

ISSN : 2259-8863

\section{Éditeur}

Presses universitaires de Rennes

\section{Édition imprimée}

Date de publication : 30 juin 2012

Pagination : 141-155

ISSN : 0183-973X

\section{Référence électronique}

Elisabeth Delattre, «Le double portrait et les trois oranges : effets de miroir dans Shamrock Tea de Ciaran Carson », Études irlandaises [En ligne], 37-1 | 2012, mis en ligne le 30 juin 2014, consulté le 01 mai 2019. URL : http://journals.openedition.org/etudesirlandaises/3037 ; DOI : 10.4000/ etudesirlandaises.3037 


\title{
Le double portrait et les trois oranges : effets de miroir dans Shamrock Tea de Ciaran Carson
}

\author{
Elisabeth Delattre \\ Université d'Artois
}

\section{Résumé}

Cet article a pour but de montrer comment, par le biais de la représentation picturale, en l'occurrence une huile sur toile du peintre flamand Jan van Eyck, Ciaran Carson a introduit dans Shamrock Tea une dimension hybride et plurielle qui fait de ce récit en prose un miroir aux reflets infiniment changeants. Certes le phénomène du double tend à remettre en question la représentation du monde et de la réalité, mais dans le même temps le concept de la Trinité, métaphorisée par les trois oranges du tableau, vise à donner une appréhension englobante du monde.

Mots clé : Ciaran Carson, Shamrock Tea, peinture, prose, représentation, double.

\section{Abstract}

This article aims at showing how, by way of pictorial representation, ie. an oil on wood by Jan van Eyck, Ciaran Carson has introduced a hybrid and plural dimension in Shamrock Tea, which transforms this prose narration into a multiple and forever changing mirror. Indeed, the concept of the double tends to question the representation of the world and reality, yet at the same time the metaphor of the trinity embodied by the three oranges in the painting purports to offer an all-including apprehension of the world.

Keywords : Ciaran Carson, Shamrock Tea, painting, prose, representation, double, time.

Like some son looking for his father, or the father for his son We try to piece together the exploded fragments.

(Ciaran Carson, « Hamlet»)

Ouvrage en prose publié en 2001, Shamrock Tea ${ }^{1}$ comporte en couverture une vision fantasmagorique d'une partie du célèbre tableau du peintre flamand Jan van Eyck, Arnolfini et sa femme ${ }^{2}$, double portrait datant de 1434 que l'on peut

1. Ciaran Carson, Shamrock Tea, London, Granta Books, 2001, 308 p. Les références à cet ouvrage apparaîtront entre parenthèses dans le corps du texte. Une traduction française est parue en avril 2004 aux éditions Actes-Sud sous le titre Thé au trèfle (traduction Sophie Bastide-Folz).

2. Ce tableau ne comportait pas de titre à l'origine. Il figure à la National Gallery sous le nom de The Arnolfini Marriage. Il est encore appelé Arnolfini and his wife ou même The Betrothal of the Arnolfini. 
voir à la National Gallery de Londres. Cette huile sur bois sert de toile de fond à cet ouvrage de fiction sélectionné pour le prix Booker, qui fait suite à un autre ouvrage en prose Fishing for Amber publié deux ans auparavant ${ }^{3}$. Shamrock Tea est à la fois un roman - l'histoire du narrateur, jeune garçon nommé lui aussi Carson - un récit historique et policier, un essai sur Oscar Wilde ou Conan Doyle, un traité sur l'art de peindre, une biographie du philosophe Wittgenstein, un livre de réflexion sur la magie noire et les drogues hallucinogènes, voire encore un pamphlet sur la politique nord irlandaise, le tout dominé par la présence récurrente de St Patrick ou celles de Dympna, sainte patronne des fous, et autres figures de la mythologie chrétienne, car " tout est lié " (" everything connects ") selon l'auteur, en somme un ouvrage inclassable ${ }^{4}$ qui fait penser à celui de Julian Barnes publié en 1985 : Flaubert's Parrot. Qui plus est, la présence, dans l'ouvrage de Carson, d'un perroquet surnommé «Vittoria ", n’est évidemment pas fortuite. Carson emprunte autant au registre du réalisme qu'à celui du fantastique et du conte oral en juxtaposant les légendes, les rêves et les mythes religieux.

La complexité du texte de Shamrock Tea ${ }^{5}$ a sans doute pu parfois rebuter ou dérouter critiques et/ou lecteurs. Elmer Kennedy-Andrews écrit à ce propos : "In a metafictional text such as Shamrock Tea, where the story has only tenuous connection with reality as it is commonly understood, the reader can all too easily get lost ${ }^{6}$. " Certes, la part de mystère subsiste jusqu'au bout, qu'est-ce exactement que Shamrock Tea? Diverses définitions sont données dans le cours du récit, mais le lecteur reste dans l'incertitude. Comme le constate par ailleurs Conan Doyle, "there was more to shamrock than meets the eye" (229). Si l'on ne peut pas parler

3. Fishing for Amber reçut un accueil parfois mitigé de la part des critiques, ainsi l'opinion d'un critique américain: "A Belfast writer much admired in Britain, Carson is known to American readers primarily as a poet. His first novel published in the United States, "Fishing for Amber", attracted little notice. But "Shamrock Tea", which made the long list for the most recent Booker Prize, could win Carson a cult following here. Like "Fishing for Amber", the new book is a magic carpet embroidered with stories within stories. "Shamrock Tea", though, is more streamlined, its humor better camouflaged and more intricately rigged " (Kenneth Baker, San Francisco Chronicle, Sunday, January 13, 2002, [http://www.sfgate.com]).

4. Lors de la parution de l'ouvrage, John Kenny écrivait à propos de ce caractère hétérogène : "Issued in Granta's neat small hardback format, Ciaran Carson's new novel is not a novel. As with his two previous prose works, The Star Factory (1997) and Fishing for Amber (1999), the publishers advise a cataloguing of the book as "Fiction"; yet to accept the applicability of that term would be to pass too easily over the intriguing intergeneric project Carson has recently adopted " (John Kenny, "My word! Carson's at it again ", Irish Times, 17 March 2001, [http://www.ireland.com/dublin/entertainment/books/bookarchive/shamrock.html]).

5. Voir l'article de Catherine Conan, «L'auteur et ses fantômes dans la prose de Ciaran Carson : Fishing for Amber et Shamrock Tea " in Gaïd Girard (ed.), Territoires de l'étrange dans la littérature irlandaise au XX siècle, PUR, 2009, p. 93-102.

6. Elmer Kennedy-Andrews, "Carson, Heaney, and the art of getting lost " in Ciaran Carson. Critical Essays, edited by Elmer Kennedy-Andrews, Dublin, Four Courts Press, 2009, p. 233. Dans un chapitre consacré en partie à Shamrock Tea, Neal Alexander conclut: "One version of reality only ever exists in tension with its possible others, and Carson's narrative imagination highlights the processes whereby identities are formed and negotiated » (Neal Alexander, Ciaran Carson: Space, Place, Writing, Liverpool, Liverpool, University Press, 2010, p. 170). 
de mystification proprement dite, l'impression reste floue, l'auteur s'ingéniant à brouiller les cartes ${ }^{7}$ pour rester peut-être maitre du jeu. Le gommage des frontières entre les genres, les textes, les époques, est un facteur de déstabilisation ontologique tout autant qu'esthétique qui trouve un outil de choix dans le maniement infini et obsédant du portrait et de son miroir. Certes le double, comme image spéculaire, est en relation directe avec le stade du miroir; il instaure également une relation formatrice et aliénante entre le sujet et la société. Le phénomène du dédoublement vise à remettre en question la représentation du monde et la réalité, on assiste alors à une mise en crise de l'histoire et du discours historiographique, car le récit historique officiel prend une tournure hétérodoxe.

On s'attardera tout d'abord sur la construction hybride et plurielle, qui fait du récit une sorte de miroir brisé. Cette partie sera intitulée " tous pour un " en référence à la décision finale de la jeune Bérénice de participer à l'aventure surnaturelle qu'est cette traversée du miroir, au voyage dans le passé auquel les trois enfants, devenus tels les trois mousquetaires d'Alexandre Dumas, sont conviés : "All for one and one for all " (249). L'analyse portera ensuite sur la déstabilisation à laquelle le récit tout autant que les figures du narrateur et du lecteur sont soumis, le texte devenant un miroir dont les reflets deviennent brouillés à force d'être multipliés, avant de conclure sur le concept de la trinité dans la représentation du temps, qui vise à donner une appréhension englobante du monde, le concept du double débouchant sur celui du triple.

\section{圈 «Tous pour un »}

Pour Carson, tout découpage représentationnel de la réalité, comme toute proposition conceptuelle, ont toujours un caractère provisoire, une sorte de chaîne reliant toutes choses $^{8}$. Le monde est à la fois unique et multiple, comme il le déclarait en 2001 lors de la parution de Shamrock Tea:

All the things of the world are like each other in some way, and the world is one thing in some sense while at the same time being a multiplicity of different things. It's up to yourself, at the end of the day, whether you agree with Wittgenstein that the world is everything that is the case plus a lot of other stuff as well - which it might be $e^{9}$.

\footnotetext{
7. C'est ce que souligne Neil Corcoran à propos de l'oeuvre en vers de Carson : « Carson's narratives [...] are, rather, exfoliations, turnings and returnings, digressions and parentheses, lapses and dissolvings, mazes of the seemingly aleatory and circuitous" (N. Corcoran, Poets of Modern Ireland, Cardiff, University of Wales Press, 1999, p. 180).

8. "For all life is a great chain, the nature of which is known when we are shown a single link of it» (12).

9. John McKeown, "Plotting the swirling kaleidoscope », The Irish Times, April 2 2001, [http://www.ireland.com].
} 
Carson échafaude son oeuvre à partir d'un réseau complexe d'échos intertextuels qui ont une double incidence, puisqu'ils fournissent l'arrière-plan esthétique de la référenciation mais aussi les outils d'un retour critique sur la représentation. Les deux épigraphes sont significatives à cet égard. L'extrait du Livre de peinture (Het Schilder-boeck), publié en 1604 par le peintre, poète et théoricien flamand Karel van Mander, désigne l'espace originel du texte, celui de la peinture, et plus précisément des couleurs qui forment l'arrière-plan de l'écriture, puisque l'ouvrage de Carson est divisé en cent un chapitres ayant chacun pour titre une couleur, dont certaines ne sont pas dénuées d'ironie ou de clins d'œil narquois, ainsi "Dorian Gray ", "Whiskey ", "Sardonyx ", ou encore "Imperial Blue ", etc. Shamrock Tea apparaît ainsi comme une sorte de palette sur laquelle l'auteur compose son écriture en forme de tableaux. La deuxième épigraphe est une célèbre citation du philosophe britannique d'origine autrichienne $\mathrm{du} \mathrm{xx}^{\mathrm{e}}$ siècle, Ludwig Wittgenstein, lequel apparaît lui-même comme personnage dans le récit où il joue le rôle de jardinier du pensionnat jésuite où est conduit le jeune Carson après sa consommation illicite de Shamrock Tea dans le bureau de son oncle Célestin. Cette citation, "The world is everything that is the case ", devient un leitmotiv, elle est mise en abyme et remise en question à la fois ${ }^{10}$. A l'orée du texte de Carson, la présence de ces deux épigraphes donne au lecteur quelques clés de lecture. L'auteur, en tant que créateur, énonce ses choix esthétiques et philosophiques et met en place des codes de référence. On peut dire qu'en cet avantposte, l'auteur " abat ses cartes ${ }^{11}$ ", quitte à les reprendre par la suite. De la même manière, la présence d'une bibliographie en fin d'ouvrage indique les "dettes" de l'auteur qui sont nombreuses et classées par catégories. Celle-ci témoigne de la vaste documentation qui sous-tend l'ouvrage. On peut y voir un "relevé des icônes de l'auteur ${ }^{12}$ ", selon Antoine Compagnon, elle a un " caractère citationnel ${ }^{13}$ ", mais aussi participe de la polyphonie du texte.

Shamrock Tea peut se définir comme un récit à tiroirs nécessitant un travail de réinterprétation et de réécriture du lecteur, car une partie du dénouement se trouve au chapitre inaugural. Le sort de Bérénice et de Maeterlinck après leur entrée finale dans le Double Portrait est révélé d'entrée de jeu, à l'insu du narrateur, information que le lecteur doit engranger dans sa mémoire : "On 20 July 1434, at the hour of tierce as told by the great Belfry of Bruges, in Flanders, two green-

10. Comme le souligne Stan Smith le terme " everything" est un mot favori de Carson : "Paradoxically, its very inclusiveness may reduce all specific things to one thing : their mere totality. Yet regularly, his deployment of the word is in contexts which emphasize evanescence, indeterminacy, volatility. [...] This is where Carson renegotiates Wittgenstein, for "everything that is the case" also includes everything which is not. " (Stan Smith, "Ciaran Carson's virtual realities ", in Ciaran Carson. Critical Essays, op. cit., p. 120).

11. Antoine Compagnon, La Seconde main ou le travail de la citation, Paris, Seuil, 1979, p. 337.

12. A. Compagnon, op. cit., p. 333.

13. Ibid. 
skinned twin children - a boy and a girl of about thirteen - materialized from a storm-grating in the town square, clothed in garments of what appeared to be frogskin.» (2) Qui plus est, la clé de l'intrigue n'est révélée que dans le dernier quart du livre, jusque-là le héros de l'action (mais aussi le lecteur) est dans l'ignorance totale de ce qu'on attend de lui. C'est en effet au chapitre 79, intitulé "Whiskey ", que le plan est révélé aux trois enfants, Carson, sa cousine Bérénice et son ami Maeterlinck, par l'oncle Célestin, plan qui n'est pas dénué d'humour :

Our plan is beautiful and simple: we will infiltrate the water of the Silent Valley with a powerful concentration of Shamrock Tea. The inhabitants of Belfast will have Shamrock Tea in their tea, in their coffee, in their whiskey; they will wash themselves in Shamrock tea, and be baptized with Shamrock Tea. They will see the world as it really is, a world in which everything connects; where the Many is One, and the One is Many. There will be no division, for everything in the real world refers to something else, which leads to something else again, in a never-ending hymn of praise. The world is an eternal story. (236).

Il s'agit d'un texte fragmenté, morcelé, lacunaire, répétitif et par ailleurs infini, ou pour le moins indéfini, mais dont aucune des parties ne peut être séparée de l'ensemble. La multiplication des registres, le décrochage des voix narratives, l'usage d'un discours indirect libre mais aussi de dialogues et de style télégraphique, l'intrusion de citations en font un texte polyphonique. La construction hétérogène du livre, mais en même temps suivie, et son enchaînement logique ont été soulignés par son auteur au cours de l'entretien déjà mentionné :

How much is planned and how much is worked over in retrospect I can't really say. It's the old matter of saying: Once upon a time there was a King in Ireland, what did he do, what did he wear, what happened to him... you invent the story as you go along. I don't know if you can say there's such a thing as a plot in the book, but I think there is at least a definite sense that one thing leads to another ${ }^{14}$.

En multipliant les fragments narratifs, les moments du récit, en les imbriquant, en les interrompant, Shamrock Tea précarise le récit afin de le démultiplier, de le relancer sans cesse. La structure circulaire accentue encore ce phénomène, puisque les derniers mots reprennent l'incipit de l'ouvrage ${ }^{15}$ : "Perhaps I will

14. John McKeown, op. cit.

15. On retrouve un écho intertextuel dans l'un des tous premiers poèmes de Carson, «An Early Bed » :

A bubble of damp

jaundiced the scrolled flowers

on the candy-striped wallpaper. (" An Early Bed ", in The New Estate, Belfast, Blackstaff Press, 1976, p. 12, poème reproduit en incipit de Selected Poems, Winston-Salem, Wake Forest University Press, 2001, p. 3). 
return one day to the world I first entered. For now, I wish to record something of it, if only to remind myself of what I am. The first things I remember are the colours of my bedroom wall paper..." (1 et 303).

L'ambiguiité entre réalité et fiction est au centre du protocole de Shamrock Tea. Cette construction par fragments laissant le sens comme libre, suspendu, peut par certains côtés se rapprocher de la forme du haïku qui a, on le sait, constitué un modèle pour la poésie de Carson. Yves Bonnefoy écrit, à propos des haïkus : "On croirait plutôt les pages d'un herbier depuis longtemps constitué, ou les pièces d'un jeu complexe, précieusement ouvragées ${ }^{16}$. "Seules subsistent en fin de compte la dynamique de la langue, l'engendrement des métaphores et des répétitions factuelles qui tissent le texte : "Image begets image " (27). Celles-ci le stratifient, dessinent un dédale de miroirs gauchis dans lesquels le dire se reflète imperceptiblement changeant, faussement fidèle à lui-même, et dont Loyola House, le pensionnat où est envoyé le jeune Carson, est emblématique. Mélange de style néo-classique et gothique, il est le labyrinthe où se perd l'imaginaire et où toute surveillance devient impossible, ce qui permet toutes les transgressions dans la discipline: "It was difficult, indeed, to tell on which floor of Loyola House one happened to be at any given time: the corridors were full of unexpected landings, the staircases of twists and turnings. Rooms telescoped into other rooms. " (121) Cet édifice symbolise les rêveries labyrinthiques analysées par Gaston Bachelard dans Les terres et les rêveries $d u$ repos. Bachelard y voit " une dimension angoissée ${ }^{17}$ " où domine la solitude. Le labyrinthe donne le sentiment à la fois d'un monde clos et sans limites dont la configuration est analogue à celle d'une pensée coupée du réel, fonctionnant en circuit fermé. On pourra y voir dans la vision de Carson une forme de représentation de la vie ${ }^{18}$.

Confronté à sa propre logique, le texte fait retour sur lui-même, sur ses propres conditions d'émergence par le biais de ces " croisements registraux ${ }^{19}$ " diégétiques et métaphoriques, dont parle Paul Zumthor à propos de la poésie orale, ce qui contribue au brouillage de l'écriture symbolisé par le phénomène du double qui apparaît de manière spectaculaire au chapitre 21 intitulé « Permanent Black » : la marque du stylo plume utilisé par le jeune Carson est un «Parker Duofold ${ }^{20}$ ».

16. Yves Bonnefoy, "Du haïku », in Entretiens sur la poésie, 1972-1990, Paris, Mercure de France, 1990, p. 134135.

17. Gaston Bachelard, La Terre et les rêveries du repos [1948], Paris, Corti, 1969, p. 211.

18. Bachelard indique en conclusion de son chapitre consacré au labyrinthe : «l'espace de l'être saisi dans sa primitivité est un couloir, un couloir où glisse la vie, la vie qui va toujours croissant, creusant. " (Gaston Bachelard, op. cit., p. 241). On pourra y voir une analogie avec l'image du puits de mine, "the mineshaft // of until» dans le poème "Overhead" (Ciaran Carson, Until Before After, Loughcrew, Gallery Press, 2010, p. 28).

19. Paul Zumthor, La Lettre et la voix, Paris, Seuil, 1987, p. 239.

20. Sans doute en raison de ses liens avec l'Empire britannique : "Parker Pens are Empire Made " (62), cette marque de stylo plume ne fait pas partie de la nombreuse collection décrite en détail dans l'ouvrage en prose, The Pen Friend, publié en 2009. 
C'est avec ce stylo offert par sa mère qui espérait en faire un écrivain - à l'image d'un grand-oncle nommé très ironiquement Augustine Joyce -, que le jeune garçon s'initie aux mystères des van Eck, lesquels auraient été trois frères selon la légende. Afin de mieux s'approprier le texte, ou de s'en imprégner, il recopie des extraits à l'aide de ce stylo auquel il attribue un pouvoir magique : «as if by magic, the words would become mine" (62).

\section{Brouillages et reflets}

En définitive il y a dans Shamrock Tea un style éminemment poétique qui brouille le jeu énonciatif, opère la confusion entre pensée et parole vive et contraint le lecteur à effectuer un travail de mise en scène pour commencer à comprendre le texte. L'étrange ${ }^{21}$ côtoie le réel ou se substitue parfois à lui, et fait du récit un texte fantastique, en ce sens qu'il y a "incertitude épistémologique $^{22}$ ", voire " hésitation ${ }^{23}$ " entre les explications naturelles et surnaturelles, entre l'étrange et le merveilleux. Il est significatif à cet égard qu'il soit fait mention de The Turn of the Screw qui aurait trouble le sommeil du père Brown, le directeur général du pensionnat Loyola House. On se souviendra que le dynamisme de ce récit fantastique le plus justement célèbre de Henry James repose sur le dialogue implicite entre le surnaturel et la folie. Le père Brown est lui-même le reflet inversé du personnage du même nom dans les nouvelles de G. K. Chesterton ${ }^{24}$ mettant en scène avec un humour décapant un prêtre catholique, détective de génie aux méthodes intuitives infaillibles.

Le texte de Shamrock Tea met en jeu des stratégies de variations et de métamorphoses, toute une mise en scène de travestissement qui constitue comme le théâtre de l'être et qui, en même temps, tend à dépasser le stade de l'illusion, ou plutôt à faire comme si l'illusion était la réalisation. Ainsi que l'explique l'oncle Célestin aux enfants, le tableau de van Eyck est un chef-d'œuvre d'illusion, « $a$ masterpiece of illusion, is it not?» (52). Le Double Portrait devient bien un procédé par lequel le transfert est possible d'un monde à l'autre, ainsi que le définit le jeune Maeterlinck au chapitre 98, "Myrrh »:

21. "When it's going well you do feel that you enter into another world, as if you're exploring that other world, it's quite uncanny. And the knowledge that, whatever you write, it's true, because it's the real world, is also uncanny" (John McKeown, op. cit.)

22. "epistemological uncertainty" (Brian McHale, Postmodernist Fiction [1987], London/New York, Routledge, 1996 , p. 74.

23. J'emprunte cette expression à Todorov : "Le fantastique est fondé sur une hésitation du lecteur - un lecteur qui s'identifie au personnage principal - quant à la nature d'un événement étrange. Cette hésitation peut se résoudre soit pour ce qu'on admet que l'événement appartient à la réalité; soit pour ce qu’on décide qu'il est le fruit de l'imagination et le résultat d'une illusion; autrement dit, on peut décider que l'événement est ou n'est pas. " (Tzvetan Todorov, Introduction à la littérature fantastique, Paris, Seuil, 1970, p. 165).

24. G. K. Chesterton, Father Brown [1910-1923], Harmondsworth, Penguin Classics, 2001, 397 p. 
One thing, sir, he said, is clear. We are all implicated in the case of Shamrock Tea, and of the Double Portrait, which might be described as a translating device. I use 'translating' according to its Latin etymology, which means to carry over or to transfer from one place or condition to another. (292-3)

Considéré comme l'alter ego du jeune Carson, son frère presque jumeau, Maeterlinck est présenté comme étant le neveu du grand écrivain belge prénommé Maurice dont on connaît l'importance du symbolisme dans son œuvre, mais aussi les essais métaphysiques, notamment un ouvrage La Vie des abeilles (1901), dont des extraits sont reproduits parfois verbatim, non sans quelques jeux de mots ici et là : " as busy as bees" (133), ou "bees on the brain" (135), ou encore "bee's knees" (225). Selon Maeterlinck, un être humain est double, et possède une existence à la fois intérieure et extérieure. On peut dire que dans l'ouvrage de Carson le double apparaît sous des formes multiples et tout d'abord par le truchement privilégié du miroir.

À bien des égards, le miroir dans le tableau de van Eyck est un chef-d'œuvre en lui-même, un véritable trompe-l'œil. Il s'agit d'un miroir convexe entouré de 12 médaillons miniature dépeignant des scènes de la vie du Christ. Le reflet dans le miroir comprend l'autoportrait de van Eyck en compagnie d'un autre homme, peut-être le témoin officiel de la cérémonie du mariage. Dans l'ouvrage de Carson, ces personnages ne sont pas identifiés, sans doute pour lui donner une connotation plus universelle et plus incertaine encore, ainsi que l'explique le père Brown : "The two figures in its looking-glass are about to enter the pictured room from another time and space. They are anyone and anybody. They could be me and you " (272). Mais cette composition illusionniste est aussi contredite par la signature ostentatoire et surdimensionnée qui dénonce l'artifice de la tridimensionnalité de ce tableau. Selon Jean Fabre, dans un essai sur la littérature fantastique, " [l]e miroir devient carrefour de miracles : le reflet peut y prendre une autonomie redoutable et le miroirécran présenter des tableaux animés du passé, du présent et de l'avenir; permettant de savoir à distance, il se fait passage entre deux mondes ${ }^{25}$ ". Le rôle " multiple " joué par le miroir est ainsi « à la mesure, et peut-être au-delà de sa charge potentielle initiale ${ }^{26}$ "; le récit du rêve de Maeterlinck est emblématique à cet égard : "As they whirled around me, I became the bees, or they became me. I glimpsed myself in the mirror: I had become a spinning wraith, composed of blue and yellow molecules. Faster and faster we spun, the colours merging, till they became a haze of green " (200). On peut véritablement parler de mise en abyme, de miroir interne " réfléchissant l'ensemble du récit par réduplication simple, répétée ou spécieuse ${ }^{27}$ ». Car le reflet dans le miroir est un double, c’est-à-dire à la fois un autre et un même.

25. Jean Fabre, Le Miroir de sorcière. Essai sur la littérature fantastique, Paris, José Corti, 1992, p. 217

26. Ibid.

27. Lucien Dällenbach, Le récit spéculaire, Essai sur la mise en abyme, Paris, Seuil, 1977, p. 52. 
Si, pour Ciaran Carson, la représentation suppose une fracture, une déconstruction, il existe aussi le sens du miroir dans ce qu'il a de plus intime dans notre imaginaire, celui non pas de refléter mais de faire apparaître, telle est la réflexion du jeune Carson :

I remember now gazing at my face sometimes in the washbowl mirror in the morning, wondering who this bleary stranger was who could not look me properly in the eye. At other times, it was as if I had been newly born and saw the world with the clear uncomprehending vision of a child. (184)

Un miroir fascine tant par ce qu'il contient, potentiellement, ce quelque chose que l'on ne peut pas voir, l'horreur de soi, de la mort, ce qui donne au récit une connotation tragique et fascinante, car pénétrer dans ce miroir implique la disparition de l'être au monde. L'angoisse ressentie par le personnage est palpable, d'autant que la précision n'est pas garantie quant à la destination de ce voyage dans le temps et dans l'au-delà : "Given that infinity of worlds, you need not deliberate about a choice of rooms. Only make the leap of faith and you will arrive at where you meant to be" (294), tel est le conseil donné aux enfants par le colonel Hay, vétéran des guerres contre Napoléon, après un détour erroné dans l'Irlande du début du $\mathrm{XIX}^{\mathrm{e}}$ siècle. En définitive " [l]a mort s'inscrit à la fois dans l'aventure narrée et dans l'absence de signification qui instaure l'ambiguïté finale ${ }^{28}$ ", puisque le doute persiste à la fin de l'ouvrage et l'interprétation est laissée au lecteur. Il n'y a pas de point final mais trois points de suspension.

Le thème du double, qui apparaît dans Shamrock Tea sous toutes ses formes peut-on dire, pose alors la question de l'unité et de l'unicité du sujet, et se manifeste par la confrontation surprenante, angoissante, surnaturelle, de la différence et de l'identité. C'est la découverte faite par le jeune Carson lors de sa lecture du "Yellow Book» :

I shut the book, trembling. For all its lacunae, its sometimes feeble grasp of chronology, its slipshod description, for all its shabbily objective voice, there could be no doubt about it: the Yellow Book was a portrait of myself. I had just looked into its crooked mirror. (184)

Le jeune garçon est une proie toute prête aux manifestations du surnaturel, "curiouser and curiouser ${ }^{29}$ ", s'exclame à plusieurs reprises Bérénice, sa cousine et compagne de jeux, à bien des égards considérée comme sa sœur jumelle. Le narrateur s'installe dans un double rôle, celui de témoin et de victime. C'est à

28. Jean Fabre, op. cit., p. 234.

29. Cette exclamation est aussi celle que prononce Alice après avoir mangé le gâteau : " "Curiouser and curiouser!" cried Alice (she was so much surprised, that for the moment she quite forgot how to speak good English). " (Lewis Carroll, Alice in Wonderland [1865] in The Annotated Alice edited by Martin Gardner, Harmondsworth, Penguin Books, 1970, p. 35). 
la fois un personnage en creux, apte à recevoir le surnaturel et les réactions du lecteur devant l'insolite. Car une épreuve attend les trois enfants avant de partir dans le temps. La tâche que leur assigne le père Brown après avoir prétexté un manque d'attention en classe, est de lire les Vies des Saints (Lives of the Saints), qui se trouvent dans la bibliothèque. Mais ces lectures mènent à d'autres puisque les enfants découvrent, au milieu de l'agencement inhabituel des livres, un album très étrange, sans titre, divisé en sections précédées de chiffres, dont la lecture stupéfait Carson car l'ouvrage contient des références précises à lui-même et à ses comparses: "a thick loose-leaf album bound in yellow cloth, divided into three sections of colour-coded pages, yellow, blue and green " (175). Cet ouvrage dont des extraits sont reproduits sur une douzaine de paragraphes apparait comme une sorte de miroir codé de la vie réelle, mais qui joue le rôle de mémoire plus véritable que celle que chaque être peut avoir de sa vie passée. C'est ce qu'explique l'Oncle Célestin, qui semble être de mèche avec le Père Brown :

The books are not mere biographies. Some passages include scenarios that failed to materialize; some describe events as they happened, but not as you remember them; and some are fictions. But all in all, the books contain more truth than other people's memories of themselves, which are constantly revised to suit their current images of themselves. (211)

Ce "Yellow Book ", littéralement le " livre jaune ", allusion évidente à Oscar Wilde, vu lui-même comme complémentaire indissociable de Conan Doyle ${ }^{30}$, devient une caricature de l'ouvrage de Carson, une sorte de mise en abyme, de reflet, de double de l'écrit lui-même. La fin de l'ouvrage comporte à la fois la clé de l'angoisse vécue jusque-là et une sorte de tremplin pour prolonger le saut dans l'inconnu. Mais le héros cherche aussi à savoir et attend avec curiosité une chose qui s'est produite et qu'il voudrait revoir. C'est ce qui le pousse irrésistiblement à passer de l'autre côté du miroir et à se fondre dans le Double Portrait, même si la première expérience relatée au début se solde par une très grosse fièvre et une incapacité temporaire à faire la distinction entre les dimensions ${ }^{31}$.

Certes la nature du Double signifie moins que sa fonction. "L'essentiel ", selon Jean Fabre dans Le Miroir de sorcière, " est que la figure du Double s'impose comme le lieu privilégié où l'altération joue à plein puisqu'elle frappe au cœur même de la psyché et de la personne ". " Je " devient un autre, cet autre qui est en moi «plus moi-même que moi ${ }^{32}$ ». Lorsque le narrateur retraverse le portrait pour réintégrer le monde des vivants, il devient un autre lui-même, presque identique à celui qu'il était en le quittant, en l'an 1959, mais avec un léger décalage puisque

30. "Wilde and Doyle, who might be said to be two sides of the same coin" (214).

31. "Unable to distinguish between dimensions" (33).

32. Jean Fabre, op. cit., p. 239. 
l'année est alors 1952, le lieu n'étant plus Belfast mais Gheel, les deux villes étant considérées comme des doubles l'une de l'autre. Une période d'adaptation devient nécessaire avant de trouver équilibre et sérénité : "I only gradually learned that it was not the same world as that I had left, although it was almost identical in most respects " (297), déclare-t-il dans le chapitre antépénultième intitulé très ironiquement "Chameleon ». Entre-temps, il a perdu ses deux compagnons de voyage, car ils s'étaient trouvés face à deux Doubles Portraits, deux portraits originaux que van Eyck, selon la légende vraie ou fausse, aurait peints, mais dont l'un a semble$\mathrm{t}$-il disparu depuis le $\mathrm{XIX}^{\mathrm{e}}$ siècle.

Dans Shamrock Tea, on assiste à la démonstration de la conception métaphysique du double, la référence à la caverne platonicienne au chapitre 60, intitulé "Dorian Gray ", faisant écho à la " caverne sombre " qui n'est autre que le cinéma d'où émergent au chapitre 54 ("Danube Blue ») les deux amis que sont le père Brown et Wittgenstein, le film cinématographique représentant pour ce dernier la réalité et non l'imaginaire. Or, chez Platon, notre monde n'est que le double et l'ombre de celui des Idées : on ne connaît pas, on re-découvre, on se souvient, car toute chose connaissable n'est que le double d'un modèle inconnaissable, l'apparence de l'être, le phénomène du noumène. Et c'est cette même explication qui apparait au chapitre 60 ("Dorian Gray") : "You have shown me a strange image, and they are strange prisoners. Like ourselves, I replied" (180). En définitive Shamrock Tea explicite l'idée freudienne selon laquelle le double ne serait en réalité qu'un aspect particulier d'une forme plus fondamentale, un élément dans un ensemble beaucoup plus vaste, constitué par la répétition du même : "Dans le couple maléfique qui unit le moi à un autre fantomatique, le réel n'est pas du côté du moi, mais bien du côté du fantôme : ce n'est pas l'autre qui me double, c'est moi qui suis le double de l'autre ${ }^{33}$. "C'est pourquoi sans doute Carson, une fois intégré dans son nouveau monde, change-t-il de nom et prend-t-il celui de son ami disparu, Maeterlinck, qui est aussi celui de son nouveau protecteur ou père adoptif, un riche marchand de tableaux nommé Henri Maeterlinck, apprenant ainsi à devenir un autre lui-même: "I learned to become another person" (297).

\section{Trinité et temps}

Le temps apparait ici comme la quatrième dimension qui régit le monde dans lequel nous vivons, le miroir étant assimilé aux rouages d'une horloge (" toothed like the cog-wheel of a clock» [272]). Le Double Portrait en est l'emblème significatif, comme l'interprète le père Brown au début du chapitre 91, «Orange Tea »:

33. Clément Rosset, Le Réel et son double, 1976, Paris, Gallimard, 1993, p. 91. 
The panel on which the picture is painted is made up of three Irish oak boards.

Inscribed on the back wall are the words, Johannes de eyck fuit hic, and the year, 1434. Add these digits and they make twelve, which is three times four. Four stands for the fourth dimension, time. (271)

La cérémonie à laquelle sont conviés les trois enfants devient une sorte de rituel initiatique présidé par Célestin, lequel les investit d'une mission dite " historique ». De manière significative elle commence par un passage par la bibliothèque du collège, dépositaire des connaissances accumulées au fil du temps : "Yet each had contributed their solitary labours to that vast community, and I felt a tremor of excitement at the part that we three were to play in rewriting the history book of Ireland" (251). Au double s'adjoint le triple ou plutôt la trinité. La clé en est donnée par le père Brown au chapitre 89 , "Oak ", dans son récit d'une rencontre qui aurait eu lieu dans un passé indéfini entre van Eyck et Saint Patrick, après avoir bien sûr absorbé une dose de Shamrock Tea. Le trèfle aux trois feuilles que lui aurait présenté Patrick, la saison étant le printemps en Hibernie, était symbolique de la trinité et de l'unicité de l'être: "These represent the past, the present and the future, which form what we call the Trinity. This means that one person can be three: the person in his memory, the person who thinks himself to be, and the person he wishes to be " (265-6).

Le triple est encore symbolisé par les trois figures du tableau de van Eyck, "l'homme, la femme et le chien ", mais aussi par les trois oranges situées à la droite d'Arnolfini, et que les trois enfants devenus les " trois silencieux " " the Silent Three " [250]) sont chargés de récupérer par l’oncle Célestin, le maître d'œuvre de ce plan scabreux. Toutefois on se souviendra, et ce détail est à la fois ironique et satirique, que ce portrait n'est pas celui que l'on peut voir à la National Gallery, car celui-là a perdu son pouvoir, ayant été déplacé de son contexte d'origine, Bruges, et soumis au regard de millions de non-croyants. Car il existe, ou plutôt existait, selon le père Brown, un autre tableau identique également peint par van Eyck lors de son voyage en Irlande. Les trois enfants doivent donc voyager dans le temps, dans le Bruges $\mathrm{du} \mathrm{Xv}^{\mathrm{e}}$ siècle pour ramener les trois oranges qui, selon les mémoires du peintre, contiendraient un concentré de ce fameux Shamrock Tea, susceptible d'affecter trois fois la population de Bruges et ses environs, laquelle à cette époque était égale à celle de l'Irlande du Nord en 1959. Ils doivent donc entrer dans le portrait après avoir bu une décoction de ce même breuvage préparé par l'oncle Célestin : "To all intents and purposes, Shamrock Tea is now a thing of the past, and exists only in the past. You must therefore go back into the past in order to retrieve the future " (236). Ainsi aura lieu la réécriture de l'histoire de l'Irlande, par ce truchement l'Irlande sera unifiée, tel est le rêve de 
Célestin. Car Célestin, lui-même fumeur invétéré de tabac et autres substances, dont Shamrock Tea, et par ailleurs possesseur d'une copie du tableau de van Eyck à l'origine de toute cette aventure, s'était investi d'une mission politique, comme il l'explique au début de ce même chapitre : "Our mission, said my uncle Celestine is clear. Ireland has been too long divided. It has been said that the border between North and South exists more in the mind than in any geographical reality; we must, therefore, alter that mind-set. Everything takes place in the brain " (235). En bon détective, le père Brown quant à lui voit une vision de l'Irlande dans les cinq voiles superposés qui couvrent l'épouse d'Arnolfini, telle Sainte Dympna, patronne, entre autres, des somnambules : "These five fifths are the five provinces of Ireland" (272). La référence faite au concept mythique d'une cinquième province, c'est-à-dire d'une vision culturelle pluraliste de l'Irlande, est sans doute battue en brèche par la comparaison de cette carte de l'Irlande "faite d'un réseau de fils sans fin " avec le corps du chien à poils durs qui figure dans le tableau. Et c'est au père Brown que revient la tâche de le démêler ou plutôt de l'entremêler davantage.

De manière générale, l'œuvre de Carson fait grand usage des nombres, on peut même dire que le nombre est forme et en ceci il rejoint encore une tradition médiéviste, issue du néoplatonisme, "en ce qu’il projette dans le monde sensible l'image d'une Idée subsistante ${ }^{34}$ ", comme le rappelle Paul Zumthor. Sans doute cette profusion de dates sert le propos réaliste de l'auteur qui lui permet d'ancrer son récit dans le fantastique et de jouer sur cette frontière incertaine, brouillée entre les codes et les genres, sans lesquelles le récit sombrerait dans le chaos. Sans doute, pour reprendre les termes de Léo Bersani, « [l] a précision de la date ne sert pas uniquement l'illusion de l'authenticité historique ${ }^{35}$ ». Elle nous permet " de rendre le vécu plus accessible à notre soif de catégories et de distinctions significatives $^{36}$ ". Dans ce contexte, les nombres "régissent l'espace et le temps, de même que l'individu, la société et les choses auxquelles sont joints l'un et l'autre ${ }^{37}$ ". Aux temps médiévaux, dans la composition du discours, tout autant que dans la scansion d'une activité, on faisait intervenir le nombre "pour l'intégrer à un ordre $^{38}$ ". Ainsi, conclut Zumthor, on assistait à la manifestation de "l'harmonie latente dans les objets, les âmes, les actes, c'est-à-dire la solidarité qui les unifie » car " le nombre ouvre un espace; il confère figurativement une dimension à l'éthique même, un mode d'existence mesurable à tout ce qui concerne le destin humain ${ }^{39}$ ». Le monde actuel, déstabilisé par la modernité, ne possèderait plus ces

34. Paul Zumthor, La Mesure du monde, Paris, Seuil, 1993, p. 402.

35. Léo Bersani, "Le réalisme et la peur du désir », in Roland Barthes et al., Littérature et réalité, Paris, Seuil, 1982, p. 51.

36. Ibid.

37. La Mesure du monde, op. cit., p. 402.

38. Ibid., p. 405.

39. Ibid., p. 406. 
notions englobantes, et la tâche des protagonistes de Shamrock Tea serait donc de tenter de les recouvrer.

Devenu bibliothécaire dans la ville flamande de Gheel, le narrateur, Carson alias Maeterlinck, atteint une sorte de paix et de sérénité, dans cette ville qui accueille les fous et les saints d'esprit sans distinction ni ségrégation, où il côtoie Sherlock Holmes et Wittgenstein. Comme il le déclare en incipit du dernier chapitre intitulé "Blank": "I have reached a kind of equilibrium in myself. This world is not perfect, but neither was the one from which I am an exile " (301). Tout se passe comme si les miroirs et les doubles fantomatiques ne fournissaient que des reflets inversés des autres, d'où, selon Clément Rosset, la nécessité de renoncer au double. "Car le miroir est trompeur et constitue une "fausse évidence", c'està-dire l'illusion d'une voyance : il me montre non pas moi mais un inverse, un autre; non pas mon corps mais une surface, un reflet ${ }^{40}$. "Tout comme Rosset voit une image du retour à soi dans la sereine plénitude qui se dégage des tableaux de Vermeer, Carson, pourrait-on suggérer, aurait la même vision à l'égard des tableaux de van Eyck. Car la peinture, ici le Double Portrait de van Eyck, permet de saisir l'immédiat dans toute sa réalité ainsi que l'explique Célestin au chapitre 17, intitulé «Oxblood»:

Painting, he said, is the art of making things real, because you have looked at things as they are. In order to paint a twig, you must look at a twig and to paint a tree you must look \& c. Only then do you bring the two things together. But you must also remember the injunction of Cennino ${ }^{41}$, that the occupation known as painting requires you to discover things not seen, and present them to the eye as if they actually exist. (50)

La jouissance de soi implique de ne plus chercher à se voir, mais bien de plonger dans ce très peu de réalité des choses quotidiennes, qui est aussi toute la réalité. « Il faut donc que le soi suffise, si maigre semble-t-il ou soit-il en effet : car le choix se limite à l'unique, qui est très peu, et à son double, qui n'est rien ${ }^{42}$. " Mais le double a aussi son côté constructif, toute histoire de double est également un Bildungsroman. Le texte permet de viser cet horizon ontologique où je deviens un autre, il emmène l'écrivain, et le lecteur, vers ce point imaginaire d'une altérité radicale en laquelle l'identité s'accomplirait, qui est celle de la lumière suprasensible : "Van Eyck duplicated with the brush the work of goldsmiths in metal and gems, recapturing that glow which seemed to reflect the radiance of the Divine, the superessential light " (63). Cette lumière permettra sans doute d'accéder à la Nouvelle Jérusalem que les

\footnotetext{
40. Clément Rosset, op. cit., p. 93.

41. Cennino Cennini, peintre et écrivain d'art italien du début du $\mathrm{xv}^{\mathrm{e}}$ siècle, auteur d'un traité relatif aux techniques et aux débats artistiques : Libro dell'arte.

42. Clément Rosset, op. cit., p. 124.
} 
héros de Shamrock Tea vont construire en Irlande, "in Ireland's green and pleasant land " (219), car ils étaient tous catholiques, même le trio formé par Conan Doyle, Wilde et le poète jésuite Hopkins pour qui l'univers est infini à qui sait percevoir sa qualité sacramentelle ${ }^{43}:$ "We are all Catholics in this together » (219). Et l'orange, dont le parfum domine littéralement l'ouvrage de Carson, pourrait bien avoir été le fruit paradisiaque originel. Telle est la réflexion de Maeterlinck : " Ghent, it was said, had invented the orangery: might the original paradisal fruit have been an orange? " (113). Les trois oranges du tableau de van Eyck symbolisent vraisemblablement la fécondité, mais dans la vision de Carson elles ont sans doute aussi une signification multiple, poétique autant que politique.

Pour Carson, il n'existe pas une façon définitive de raconter une histoire ${ }^{44}$ et « la littérature n'est qu'un autre élément dans un univers de discours ${ }^{45}$ " qui est le nôtre. Loin de se développer logiquement, l'ouvrage de Carson ne cesse de miner son propre discours, de se ramifier pour rejoindre de précédents rameaux. L'ouvrage se tourne sur lui-même, plonge dans son propre réseau métaphorique qui devient peu à peu sa seule échelle référentielle. Dès lors, le livre ne représente plus la réalité mais contribue à la créer, à lui donner forme, tout en s'en démarquant. Même si le monde de Shamrock Tea est un monde fascinant et envoûtant pour le lecteur, une sorte de Tea Party à la Lewis Carroll, il n'en reste pas moins un univers inquiétant et sombre, où le plus fou n'est pas celui qu'on pense ${ }^{46}$. Ce monde deviendra " a minefield salted with eyebright ${ }^{47}$ " dans un recueil de sonnets publié en 2009 sous le titre On the Night Watch, avant que le poète, dans Until Before After publié l'année suivante, ne transporte son lecteur, "over threshold // after threshold", " to our room // full of light ${ }^{48}$ ".

43. "If the doors of perception were cleansed, everything would appear as it is, infinite" (227).

44. "There is no final way of telling a story " (R. Brandes, "Ciaran Carson interviewed ", The Linenhall Review, 8, 1990, p. 84).

45. "Literature is just another element in a universe of discourse " (Frank Ormsby, "Ciaran Carson interviewed ", The Linenhall Review, 8:1, 1991, p. 6).

46. Je fais référence aux paroles de l'auteur lors de l'interview déjà citée : "It's about madness at the end of the day. You could say that the whole book is about somebody who is actually insane. He's even in hell maybe [...] But at the end of the writing you're not sure which is the real world: the one you've written or the one you physically inhabit. The world in Shamrock Tea is a curious, interesting world - I hope-but it's not all pleasant, there's a definite dark side to it" (McKeown, op. cit.).

47. Ciaran Carson, On the Night Watch, Loughcrew, Gallery Press, 2009, p. 14.

48. Ciaran Carson, Until Before After, op. cit., p. 119. 